are of the bladder through the perineum is always necessary. I believe also when you have a fistula it would be better to drain the bladder through the perineum.

What part of the operation is the most difficult? In the first place getting the mucous membrane clear to the end of the glans penis. It is nearly always impossible to find sufficient mucous membrane to reach to the end of the glans peuis. While the urethra can be drawn out surprisingly, it also retracts afterward somewhat surprisingly. I am sure that Dr. Parham is entitled to great credit for the suggestions he has made. I believe in the case I operated on, if I had separated the corpora cavernosa further back, I would have had a better result, and this fistula would probably not have occurred. The third point is that the formation of the canal from the point where the abnormal meatus is situated is somewhat difficult. However, I believe from the success of this operation in my own particular ease, and from these other operations that have been reported, that it is very much superior to any of the other operations that have been proposed.

\section{TUBERCULOSIS OF THE MAMMARY GLAND.*}

\section{A. H. LEVINGS, M.D. MLWWAKEE, WIS.}

Dr. Scudder, ${ }^{1}$ in an exhaustive article on "Tuberculosis of the Mammary Gland," states that an examination of the literature showed only eighty cases reported up to that time, twenty-three of which were rejected on account of imperfect data. This list, however, does not include twelve cases reported by Albert in his work on surgery.

Tubercular infection is so frequently met in the various tissues and glands of the body that it seems unusual that only fifty-seven well authenticated cases of tuberculosis in the mammary gland should have been reported up to 1898. It is, of course, to be expected that not all cases diagnosed as tuberculosis of this gland were reported, but had they been, the number would still be small, and the cases reported since 1892 are so few that we are forced to believe that the disease often goes unrecognized.

It is conceded that tuberculosis of the mammary gland is most frequently found in young women between the ages of 20 and 35 , or during the period when the gland is functionally most active. A few cases have been reported as occurring before and after menstrual life. Roswell Park reported a case in a girl 12 years of age, and Mr. Payne saw an infant with a tubercular abscess of the breast, following which there was general tuberculosis and death. Several other cases have been reported at ages under 20. Cases have also occurred in women after 50 years, and even after 60 years of age. The condition is occasionally observed in men. In Dr. Scudder's eighty cases six were males, and in forty cases reported by Mandry one was a male. It is said that more than one-half of the women affected have borne children, and that in a considerable number of these the process has originated during pregnancy.

It is held by the great majority of writers, and this I believe to be true, that the process is to be observed most frequently in spare, weakly, anemic individuals, in those who have inherited the so-called scrofulous diathesis or tubercular predisposition, and especially in such as have a parent or near relatives who have or are suffering from tuberculosis. Tubercular infection of the mammary gland may be primary or secondary. When primary it may occur through the milk ducts or through

* Read at the Fifty-fourth Annual Session of the American Medical Association, in the Section on Surgery and Anatomy, and upproved for publication by the Executive Committee: Drs. $\mathbf{A}$. J. 1. Amer. Jour. Med. Scl., July, 1898. abrasions of the nipple or of the skin covering the breast. It is thought that the great majority of cases are secondary to a tubercular process in the lymphatic glands of the axilla or neck, or to one in an adjacent rib, pleura or lung. The focus, however, may be situated in distant bones, in the genito-urinary or intestinal tract, in the peritoneum or skin. When secondary and the foci are situated more or less remotely, infection may occur, and frequently does, through the arterial circulation. Infection undoubtedly takes place, however, in many of these cases through the lymphatic circulation by a retrograde process. Retrograde infection or infection carried against the lymph cur rent is seen every day in cases of carcinoma. A case of epithelioma of the nipple came under my observation in which there were metastatic growths in the membranes of the brain, these having taken place through the lymphatic circulation. It is also of frequent occurrence in carcinoma of the mammary gland to observe enlargement of the lymphatic glands of the neck. Although it is held that the bacillus of tubercilosis is non-motile, it is probably true that these bacilli are often carried against the normal lymph current by cellular action or by reverse currents. Infection may also occur by contiguity of structure in cases where the submammary tissues, the adjacent ribs, or parietal pleura are implicated. If infection occurs through the milk ducts, the process must be situated primarily within the ducts or the acini. If the bacilli reach the breast through the circulation, lymphatic or arterial, they would naturally be arrested in the tissues directly surrounding the alveoli, as here the arteries which supply the gland break up into a network of capillaries, while the lymphatic radicles take origin within and about the lobules of the gland. When infection occurs through contiguity of structure the connective tissue of the gland will be primarily implicated.

\section{THE ANATOMY}

It might be well in this connection to review briefly. the anatomy of the lymphatics of the chest for the purpose of determining the possible channels of infection to the mammary gland through these vessels. It is generally held that the lymphatics in the mammary gland of the female are more highly developed than they are in any other gland in the body. They are generally divided into two groups, the superficial, which passes to the axilla, and the deep, which follows largely the branches of the internal mammary artery and goes to the anterior mediastinum. The axillary glands may be divided into three chains. The first, or superior, lies along the lower border of the pectoralis minor muscle, and is in relation with the long thoracic vessels.

It receives-most of the lymphatics from the upper and outer portion of the mammary gland, the front of the chest and from the abdominal wall above the umbilicus. The second or middle chain is situated in the adipose tissue of the axilla and on the inside of the axillary vein. It receives the lymphatics largely from the upper extremity, and the efferent vessels from both the superior and posterior chains. The posterior group lies along the posterior border of the axilla in relation with the subscapular vessel. It receives the lymphatics from the scapular region of the back and from the inner and lower quadrant of the breast. The deep lymphatics of the gland anastomose freely with the superficial and with those from the pectoral fascia. Some of these vessels from the outer side go to the axilla, while others accompany the intercostal vessels and enter the 
right lymphatic or left thoracic duct. The deep lymphatics, which originate in the inner hemisphere of the breast and in the inner portion of the pectoral muscles, penetrate the second, third and fourth intercostal spaces and communicate with the glands, six or eight in number, which are situated along the internal mammary artery.

\section{LYMPHATICS OF THE LUNGS.}

There are two sets, the superficial and deep. The superficial is placed beneath the pleura as a minute plexus covering the outer surface of the lung, communicating freely with the pleural cavity, and invading the interlobular tissue. It terminates at the root of the lung in the bronchial glands. The deep set commences in the mucous lining of the bronchial tubes and accompanying the ressels terminates in the pulmonary lymphatic glands situated along the walls of the small bronchi and in the angles of their subdivisions. There are about forty glands in the right lung and thirty in the left. Efferent vessels from these pulmonary glands join with the superficial lymphatic vessels and end in the bronchial glands. The efferent vessels from the bronchial glands communicate with the mediastinal glands and traverse the tracheal and esophageal glands, terminating on the right side in the right lymphatic, and on the left in the thoracic duct. There may be, then, a direct circuit of lymph by retrograde action from the neck and axilla to the breast, and from the lungs, pleura and pulmonary and bronchial glands through the mediastinal glands and their lymphatics along the branches of the internal mammary artery to the breast. The arteries which supply the breast are from the thoracico-acromialis to the upper lobes, from the long thoracic and external mammary to the outer lobes, and from the anterior intercostal and perforating branches of the internal mammary to the inner lobes. Each gland consists of from fifteen to twenty tubo-racemose glands bound together by connective tissue and united into a hemispherical mass by adipose tissue. Each lobe has its own excretory duct. The acini, either sacular or in the form of tubules, are grouped together to constitute lobules. They are limited by a distinct membrane and lined by either columnar or polyhedral epithelial cells. The blood vessels run mostly in the superficial tissues radiating toward the areola, and from these vessels branches penetrate into the glandular mass and pass between the lobules, giving off twigs which break up into capillaries enclosing the alveoli. Naturally, then, if the bacilli are brought by the blood vessels to the gland they would become implanted on the alveoli or they might pass through their walls into the interior. The latter unquestionably may occur, and does during lactation. If the bacilli are arrested in the capillaries the tubercular process set up would be an interacinous one. If they pass into an acinus or duct and become arrested the infectious process would be within the acinus or duct. The lymphatics also run in the connective tissue stroma of the gland and commence as a network of capillaries within and around the lobules. Their radicles have practically the same distribution as have the capillary blood vessels. In consequence of a retrograde action in the lymph current the bacilli would also become implanted about the acinous wall, or possibly carried into its interior.

\section{PATHOLOGY.}

The bacilli being implanted within the tissues of the gland set up an irritation which is followed by the usual pathologic change. Round cell infiltration occurs with the formation of epithelioid and giant cells. The bacilli are often to be found in the exudate, while tubercles or tubercular masses with caseation or pus formation often occur. It is characteristic of those cases which remain for a long time as hard nodes for the microscope to show the formation of a large quantity of interacinous connective tissue. In a considerable number of my cases some of the hard nodes were made up largely of dense fibrous tissue situated between sparsely distributed acini. In these cases there will be areas of distinct caseation or those in which giant cells are to be found or the bacilli of tuberculosis and even in some in which these conditions are not demonstrated the axillary glands will show caseation and the bacilli of tuberculosis. In cases in which the infection is directly through the milk ducts or glandular structure, the process, aside from its situation, will be much the same as before with the exception that in a considerable number of these pus may be forced from the abscess cavity by pressure along the ducts and from the nipple.

\section{SYMPTOMS AND COURSE.}

The clinical picture presented by a tubercular process within the breast is a variable one. It will depend largely on the stage of the disease during which the examination is made, on the number of foci primarily infected, and also on the fact as to whether the process is primary or secondary. In many of these cases, as can be readily appreciated, the primary disease will be of such importance or severity that the condition of the breast, even if severe, will be of little or no moment. The patient's attention may first be called to the process by pain which is usually early experienced, pretty continuous and has a dull, heavy, aching or burning character. Not unfrequently, however, the pain is scarcely noticeable, and the patient's attention is first attracted to the breast in consequence of finding therein a node or nodes. These nodes, and there are usually more than one, are reasonably hard, not very sensitive and quite irregular. They can not be distinctly separated from the glandular structure, and ordinarily vary in size from that of a hazel nut to half an inch or an inch and a half in diameter. They are very seldom round, but are flat or have a very irregular contour. These nodes are often distinctly separated, but still lie in the same quadrant of the breast. The fact of their being multiple nodes is a characteristic of the disease. The gland as a rule is not enlarged, and on simple inspection nothing may be seen. The axillary glands during this early stage are enlarged and may be palpated. As the condition progresses the nodes increase in size, though very slowly, possibly coalesce, become more elastic, and finally impart a sense of fluctuation. They may become adherent to the skin and the contents of the abscess be discharged through a fistulous tract. Occasionally several fistulæ are formed in consequence of the breaking down of many nodes. While ordinarily the gland is not enlarged during the first stage of the disease, later, if pus in any considerable amount is formed, the breast becomes noticeably enlarged. If the process is situated near the posterior surface this may become attached to and quite immovable from the pectoral fascia. The nipple, in a goodly number of cases, is retracted.

\section{DIAGNOSIS.}

The diagnosis will depend on the antecedents and appearance of the patient, the history of the case and the physical examination. The antecedents will often show scrofula or tuberculosis. The appearance is often that of a thin, anemic, weakly woman of lessened resist- 
ance, while the history may establish the probability of tubercular processes in other parts of the body. The examination will usually disclose, if made before the formation of an abscess, one, more frequently two or three, quite hard, very irregular, well defined, not specially sensitive nodes which are inseparable from the glandular structure. The axillary glands are enlargedit is stated in two-thirds of the cases-and the nipple often retracted. When pus has formed the use of the hypodermic needle and the microscope will materially aid in establishing the diagnosis. When fistulæ have formed, the process being chronic, the diagnosis is easy. The process must be differentiated from scirrhus, actinomycosis, benign growths and from cysts due to chronic inflammatory processes. In scirrhus the patient is usually more than fifty years of age. The node is single and harder than in tuberculosis. The history of the cases and the cachexia are different. In actinomycosis the thickened, indurated, leathery skin, the yellow granules and the use of the microscope will establish the diagnosis. In benign growths, such as fibroids, the fact that they are usually single and distinctly encapsulated without enlargement of the axillary glands, will be sufficient to distinguish them. Multiple cysts, especially when of small size and due to a chronic inflammatory process, present the greatest difficulties perhaps in the way of diagnosis. Many of these small cysts are not to be differentiated by touch from solid nodes. In these cysts, however, the axillary glands are not usually enlarged, the history of the case is often different, and the use of the hypodermic needle will afford valuable assistance.

I have been able to find scarcely more than a dozen cases reported up to date in the United States, and believing that very many of the cases of chronic mastitis and most of those of chronic abscess of the breast are really tubercular, to say nothing of those cases in which there are distinct nodes running a chronic course, I am forced to the conclusion that in the past there has been in the great majority of cases of tuberculosis of the mammary gland a failure to make a diagnosis.

\section{REPORT OF CASES.}

Seven cases of tuberculosis of the mammary gland, all in females, have come under my observation during the past two years. Five of these patients were single. Of the two who were married but one had borne children. In three cases both breasts were affected.

CASE 1.-The first patient was 35 years of age and single. She had noticed for a year and a half an enlargement first in the right and then in the left breast. In placing the palm of the hand flat against the gland many small indurations could be felt. These seemed of considerable size when the breast. was pinched up between the thumb and fingers. The nodes were hard, irregular and reasonably well defined, but seemed to be a part of the glandular structure. The axillary glands on both sides were implicated. The nipples were not retracted. The patient's condition, aside from this, was reasonably good.

Both breasts and axillary glands were removed. The breasts were found dotted with small circumscribed abscesses, which in some cases were directly connected with the ducts and in which the bacilli of tuberculosis were demonstrated to exist. Patient made a. good recovery. In this case there was nothing in the patient's history or antecedents indicative of tuberculosis and there were no other foci anywhere in the body which could be detected.

CASE 2.-An unmarried woman, 30 years of age, with a predisposition to tuberculosis, noticed seven months previous to examination, a gradually increasing, hard swelling in the right axilla. Latterly the swelling had occasioned such extreme pain in the right arm that it was searcely endurable. During the past two months several small, hard, well circumscribed indurations could be felt within the breast structure.

The breast was excised, and with it the major portion of the pectoral muscles. An abscess of considerable size situated in the axilla and adherent to the pectoral muscle was unavoidably broken into. A large number of axillary glands were so intimately adherent to the axillary vein that excision of a portion of the vein was necessary. This was done after double ligation with catgut. The glands and abscess were causing severe pressure on the brachial plexus, some of the smaller branches of which were excised with the abscess. The breast was found on multiple incision to contain a large number of small collections of pus, many of which were not larger than a pea. Some of these were in direct connection with the ducts. The patient had never complained of anything more than an occasional burning pain within the breast.

Patient's family history was as follows: Her father was drowned, mother died of pneumonia at an advanced age, an uncle and a cousin died of consumption. There were three children, all of whom are living. The glands of the axilla showed numerous tubercular bacilli as well as many points of caseation. The process in the breast in this case was evidently secondary to the infection of the axilla.

CASE 3.-Mrs. R., aged 40, mother of three children. Very spare in figure, anemic, presents the appearance of one having but slight physical resistance. In her family history a relative of her father was supposed to have died of tubercular peritonitis, while a brother had his leg amputated for tuberculosis of the bone. After the birth of her third child eight years previously she suffered from a mammary abscess, and has been since in poor physical condition. For the past few months the patient had noticed a small, hard lump in the left breast. At the time of the examination there were two very noticeable, quite hard, reasonably well-defined nodes which evidently were inseparable from the glandular structure. One was in the upper and the other in the lower quadrant of the left breast. In the right breast there were also two or three indurations. From one of these, when pressure was made, there was a discharge from the nipple. The nipples were not retracted. The glands in both axilla were enlarged.

The breasts and axillary glands were removed. The pathologic findings showed that the glands were tubercular. The patient, one year after the operation, shows indications of pulmonary disease.

CASE 4.-An unmarried woman, aged 31 , had been for several years in delicate health in consequence of a chronic uterine and ovarian inflammation. She had worked hard as a nurse and was thin in flesh, debilitated and anemic. Her family history shows the father and mother to be living and well. There is no tuberculosis in the immediate family. $\dot{A}$ brother of the father and a sister of the mother, however, died of consumption. For four years patient has noticed a gradually-increasing enlargement in the right axilla. For a few months there has been some soreness and hardness in the right breast with slight fever. The axillary glands have caused the patient a good deal of pain. In the breast there is an illy-defined, not well circumscribed induration situated in the upper quadrant. If the breast is picked up between the thumb and fingers there is a marked induration.

The breast and axillary glands were removed. The breast, on incision, showed that the hardened area had undergone caseous degeneration. The axillary glands were found to extend along the vessels filling more or less the axillary space as far as the clavicle, making a section of the pectoral muscles necessary in order to remove them. Many of the axillary glands were in a state of caseous degeneration, and contained bacilli in large numbers. The patient, since the operation, has shown decided improvement, and although at times annoyed by cough she has gained in flesh and strength.

CaSE 5.-A single woman, aged 44. Father and mother lived to be old. No tuberculosis in family. Patient had a severe pulmonary hemorrhage twenty years ago and a second one year ago. Both from the right lung. In the right apex there is prolonged expiration with much rattling and a decided dull- 
ness. There is considerable cough and some expectoration. The latter contains tubercle bacilli. During the last eight months there has appeared in the left breast, just above the nipple, a mass two inches long .by half an inch in thickness. This area is quite hard, and from it several' strands pass out to the border of the breast, while two or three run in toward the nipple. The axillary glands are enlarged. Patient, al. though having some continued fever, looks comparatively well. lung.

Operation was not advised on account of the condition of the

CASE 6.-Female, aged 30, of spare figure, is anemic and not strong. Never, however, has suffered any serious illness. Family history good. For several months she has noticed hardened areas in both breasts. The condition has caused a good deal of anxiety and the patient has apparently lost flesh and strength. Has no cough. On examination there are several indistinct and separate hardened areas in each breast. Nipples not retracted. Glands in both axillæ enlarged.

Axillary glands and breasts removed. Sections show hardened areas in the breast and the axillary glands to contain caseous material in which the bacilli of tuberculosis are found.

CASE 7.--Female, aged 36. Married nine years; never pregnant. Spare in figure, weak and anemic. Never has been ill. Family history good. She has noticed in the right breast more or less of pain for the past three months, also a swelling, which has gradually increased in size. At the time of exam. ination there was a reasonably soft, fluctuating growth the size of a hen's egg situated in the upper and outer quadrant of the gland.

The mass was only mildly sensitive on examination. There Were also several small indurations in the breast. The axillary glands were distinctly enlarged. Patient's temperature was not above 99. In the outer quadrant of the left breast.there was also a soft, not well-defined and fluctuating mass as large as a small orange, while directly above the nipple were two hard and not well-defined indurations. Glands in both axillæ were enlarged.

The patient refused to have the breast entirely removed, consequently free excisions were made of the fluctuating masses, these excisions being made to include the indurated areas. In cutting across the ducts many of these were seen to be in intimate relation with the abscess cavities and to contain pus. The pus in both cavities was found on microscopic examination to contain the bacilli of tuberculosis.

Of these seven cases two were secondary to tuberculosis of the axillary glands, while one was secondary to tuberculosis of the lung. Of the six cases operated on fire seem to have completely recovered.

\section{TREATMENT.}

This will depend largely on the general condition of the patient and the extent of the disease in the breast. If the process in the gland is secondary to a severe and non-curable condition situated elsewhere in the body nothing more than palliation should be practiced. If the infection in the gland is secondary and the primary focus not severe or amenable to treatment, then there should be an effort made to eradicate the infection in the gland. If there be but a single collection of pus without adjacent nodes, indicating but a single focus of infection, the abscess may be incised, washed out thoroughly, curetted and drained. This course has been successfully practiced, and is recommended by Warren. If multiple foci of infection exist, and they are confined to a single quadrant of the breast, this portion may be excised. It is, however, the better practice where dissemination has occurred to excise the entire breast. If the axillary glands are implicated, as they usually are, they should be removed.

\section{CONCLUSIONS.}

Tuberculosis of the mammary gland is not an unfrequent condition, but one which goes unrecognized. The process may remain for months, and even years, in the form of hard, irregular, reasonably well circumscribed nodes. Liquefaction and pus formation are secondary and later processes. Tuberculosis of the breast occurs with especial frequency during menstrual life. It occurs with greatest frequency in single women. The condition is more often primary than has been generally supposed. The tubercular infection often implicates both glands nearly simultaneously. Palliative treatment may be practiced when the process is circumscribed. When the processes are diffuse radical measures should be recommended.

\section{DISCUSSION.}

Dr. B. B. DAvis, Omaha-A case under my observation had a very large tumor and the axillary glands were considerably involved. At one point in the breast there had been a perforation and fistula with a history of considerable material being discharged, which under microscopic examination was demon strated to contain tubercle bacilli. In the other case, I think I should have erred had I not recently read a very able article by a German operator, in which he discussed in detail the operation. This was in a comparatively young woman, about 30 years of age, if I remember rightly. Although there seemed to be one nodule, it was separate and $I$ found on examination that it consisted of three different nodules, aggluti. nated and in a state of degeneration. All I did was to excise that portion of the process containing the nodules. There was no enlargement of the axillary glands and the patient recovered. In the other case I did the same kind of an opera. tion that I should have done if the case had been carcinoma of the breast.

Another point is in regard to the temperature. In these tubercular cases which have existed for any considerable length of time we nearly always have some rise of temperature in the evening, and frequently subnormal in the morning, and this was particularly characteristic in the first case I men. tioned. I have been under the impression that a good many of these cases in which there is a hard nodule of the breast and the breast is removed, supposedly as a carcinomatous case, are in reality tubercular, and $I$ believe tuberculosis of the mammary glands is a much more common condition than is gen. erally supposed.

Dr. T. J. Sullivan, Chicago-There is no reason why tuber. culosis should not be found in the mammary gland, just as it is found in every other portion of the body, and I believe that there is no tissue of the human body that is not liable to be infected. I think we can even go further and recall the statement of some of the authors that the tubercular germs in a greater or lesser number exist in all our bodies and that sooner or later we may, any of us, bave it. That being true, we may find the tubercular germs in any portion of the body.

I had a case of this condition occurring in a woman, of average health, mother of ten children, who.had always enjoyed good health, and had been compelled to work hard to maintain the family. She had had swelling in the breast, appearing about six months previously, after working hard. Previous to presenting herself to me for examination she had been exam. ined by a physician, who informed her that she had carcinoma. The appearance would indicate that she was suffering from carcinoma. I placed my hand on the breast im. mediately over the tumor and detected a small, hard nodule. In presenting her to a class of medical men the following diagnosis was made: Retraction of the nipple; hard nodule; no axillary enlargement; healthy and well; no pain.

She was kept under observation for a couple of weeks, at the end of which time the nodule had enlarged slightly. I then was in considerable doubt as to the nature of the tumor and removed a small wedge under local anesthesia. Pathologic examination of this wedge revealed the fact that it was due to an inflammatory process. I then decided to remove the whole breast in a radical operation, including the axillary glands. I did so, and the appearance of the nodule presented to my mind the appearance of scirrhus of the breast, 
and a good pathologist who was present at the observation said that undoubtedly it presented all the appearances of scirrhus. The operation was complete, however, without further attack on the axillary glands, and subsequent pathologic examination revealed marked tuberculosis, which healed rapidly.

It is time to call attention to this subject and give these patients the advantage of early operation.

Dr. William Knight, Hartford, Conn.- -Just a little criticism of one point in regard to abscess. Dr. Levings said you could open the tumor and drain thoroughly. The tendency in treatment of those cases is to prevent absorption. It seems to me that it is folly to interfere with it. It also seems to me much better to open it and drain thoroughly with plenty of absorbent cotton. I think we will get much better results by the treatment of abscess in this manner.

Dr. ANDREw W. Morton, San Francisco-I have had experience with only one case which puzzled us considerably. We used the tubercular serum to make the diagnosis, and got the characteristic reaction which we usually get in using this serum where there are tubercles. After the removal of the gland the microscopic examination showed the diagnosis to be correct.

Dr. A. H. Levings, Milwaukee-I may add that one additional case has come under my observation since this article was written. Of my cases of tuberculosis of the mammary gland one was secondary to a tubercular process of the lungs: while two were secondary to tubercular processes in the axilla. The others, so far as I was able to determine, were primary within the breast itself. In some of these I think the infection was through the milk ducts. In others the infection was through the circulation, arterial or lymphatic. I am very confident that we have been mistaking these cases for carcinoma because the two conditions may present practically the same symptoms, in that there is a hard nodule, retraction of the nipple and enlargement of the axillary glands.

In regard to the treatment of opening and curetting an abscess, I am of the opinion that in a case in which there is but a single abscess with no out-lying nodes, this treatment may be recommended, and probably will, if properly carried out, result in a large number of cures. This, in fact, is the treatment generally which we are accustomed to follow in cases of tubercular abscess.

\section{ABSCESS OF THE SPLEEN.* WALTER M. SPEAR, M.D. ROCKLAND, ME.}

Abscess of the spleen is rare, and when a case falls into our hands and is operated on successfully, it is worthy of record.

About the middle of May, 1901, I was called to see Mr. A., and obtained the following history:

History.-American, age 74 years, weight 211 lbs. Family history: Mother and one sister died of consumption. Father died of some brain trouble. Previous history: Has had two severe falls, the last one two years ago, from which he never fully recovered. Jan. 10, 1901, was seized with a severe attack of influenza, which completely prostrated him. At the end of three weeks the temperature and pulse were normal. He bas never lived in a malarial district and has never suffered from typhoid or malarial fever. Since the beginning of his sickness he has constantly lost in weight.

Present Illness.-Patient in bed, anemic, covered with a cold perspiration, very nervous, anorexia, night sweats, slight constipation. Complains of numbness in legs and feet, slight though constant pain in left thorax just above lower border of ribs. At times be is pressed for breath, which appears to be due to his nervous condition.

Physical Examination.-Temperature normal, pulse 100, weight about 175 lbs., heart's action-very feeble, slight tender. ness on deep pressure under lower border of ribs on left side,

- Read at the Fifty-fourth Annual Session of the American Medical Assoclatlon, in the Section on Surgery and Anatomy, and approved for publication by the Executive Committee: Drs. A. J.
Ochsner, J. E. Mocre and De Forest Willard. reflexes normal. I thought that his condition was due to the severe attack of influenza which he experienced. Gave him strychnin and tonics. The improvement in his condition was marked, so much so that in a few days he was up and downstairs. I did not see him again. until May 30, 1901. His physician then stated to me that the patient, while being carried upstairs on his son's back the night before, cried out that something had given way in his left side and that he was very faint. He was immediately put to bed and stimulants administercd, to which he at once responded. I saw him the next morning and found his pulse 64 , and temperature normal. He was very nervous, had a sallow cachectic look, and had lost a great deal of flesh. He complained of constipation, and of severe pain in left hypochondrium. Some edema of legs and ankles was present. His heart was much stronger. Hard tumor in left side extending above lower border of ribs, tender, no fluctuation, urine negative. I considered the tumor malignant, which diagnosis was coincided in by several physicians. Advised exploratory laparotomy.

operation.-June 8, 1901. On examination under ether I found a hard, non-fluctuating tumor extending from above the lower border of the ribs downward to the crest of the ilium and inward to the linea alba, smooth, most prominent just below ribs. Incision made over most prominent part of the tumor. Found an enlarged spleen very dark in color, which was bound down by numerous adhesions. The anterior surface was comparatively free. Anterior wall of abscess thick, posterior wall very thin, indicating that it would have soon ruptured, allowing contents to escape into abdominal cavity. Because of the thinness of the wall and the numerous adhesions $I$ considered an attempt at removal dangerous. Walled off with gauze and made an incision into the spleen through which was evacuated two quarts of creamy pus mixed with a little blood and cheesy-looking material. Cavity curetted, washed out and spleen sutured to abdominal wall with chromicized catgut, iodoform gauze drain. Convalescence rapid and uneventful and in five weeks he was up and dressed. On Aug. 2, 1901, he suddenly became unable to swallow. Aphasia and hemiplegia of the right side appeared, and after this he rapidly failed and died Aug. 4. 1901. Unfortunately an autopsy was not obtained.

Report of Dr. C. D. Smith, who made an examination of the pus: "T fail to find tubercle bacilli, yet the presence of calcareous material is strong evidence of that nature of the abscess. It contains a multitude of lymphoid cells, much pus, a few crystals of hematoidin showing that there has been a hemorrhagic area in the organ or an embolus, and a ferv bits of characteristic yellow elastic tissue and fragments of spleen tissue."

Several cases of abscess of the spleen have been reported in which the pus was sterile, and from the absence of any previous septic condition, and the constant normal temperature, I am inclined to think that it was sterile in this case.

I ought to have made a correct diagnosis, but was led astray by the absence of fever, the slight pain, the rapid emaciation and extreme constipation. A blood count would have been of great assistance. Bessel-Hagen thinks the considerable increase of leucocytes in the blood of the greatest diagnostic significance.

The condition in this case was probably due to an embolus lodging in the splenic vessels which, on account of the slowness of the blood-current in terminal arteries, are peculiarly susceptible to the lodgment of emboli.

\section{SUMMARY OF THE IITERATURE.}

In looking over the literature on this subject, I have collected 65 cases, 23 of which occurred after an attack of malaria or typhoid fever, 1 after yellow fever and 3 after appendectomy. The others were ascribed to various causes, such as trauma, disease of heart, sudden chilling of the body after excessive heat, tuberculosis, anthrax, alcoholism and exhaustion. Eight cases in which the 